\title{
Using Pressure Map Sequences for Recognition of On Bed Rehabilitation Exercises
}

\author{
Ming-Chun Huang, Student Member, IEEE, Jason J. Liu, Student Member, IEEE, Wenyao Xu, Member, IEEE, \\ Nabil Alshurafa, Student Member, IEEE, Xiaoyi Zhang, Student Member, IEEE, and Majid Sarrafzadeh, Fellow, IEEE
}

\begin{abstract}
Physical rehabilitation is an important process for patients recovering after surgery. In this paper, we propose and develop a framework to monitor on-bed range of motion exercises that allows physical therapists to evaluate patient adherence to set exercise programs. Using a dense pressure sensitive bedsheet, a sequence of pressure maps are produced and analyzed using manifold learning techniques. We compare two methods, Local Linear Embedding and Isomap, to reduce the dimensionality of the pressure map data. Once the image sequences are converted into a low dimensional manifold, the manifolds can be compared to expected prior data for the rehabilitation exercises. Furthermore, a measure to compare the similarity of manifolds is presented along with experimental results for five on-bed rehabilitation exercises. The evaluation of this framework shows that exercise compliance can be tracked accurately according to prescribed treatment programs.
\end{abstract}

Index Terms-Isomap, local linear embedding, manifold learning, pressure images, range of motion, rehabilitation exercise.

\section{INTRODUCTION}

$\mathbf{I}^{\mathrm{N}}$ $\mathrm{N}$ traditional medicine, physical rehabilitation programs are performed in hospitals or outpatient treatment centers, where therapists trained in rehabilitation provide guidance as well as monitor patient recovery. Rehabilitation exercise programs help patients return to regular health after surgical procedures or illness.

Physical rehabilitation is well recognized to provide accelerated and long lasting benefits to patients [2]. Johns Hopkins Hospital implemented physical rehabilitation programs in early postsurgical stages, and found up to $22 \%$ reduction in ICU stays and clear reductions in net financial costs [3]. The aim of such rehabilitation regimens is to improve muscular strength and specific body range of motion. Patients undergoing physical rehabilitation follow the exercises assigned by physical therapists on a regular or semiregular basis. There is a need for physical therapists to manually monitor and evaluate the rehabilitation process

Manuscript received June 15, 2013; revised September 4, 2013 and December 16, 2013; accepted December 20, 2013. Date of publication January 2, 2014; date of current version March 3, 2014.

M.-C. Huang, J. J. Liu, N. Alshurafa, X. Zhang, and M. Sarrafzadeh are with the Wireless Health Institute, Department of Computer Science, University of California, Los Angeles, CA 94607, USA (e-mail: mingchuh@cs.ucla.edu; jasonliu@cs.ucla.edu; nabil@cs.ucla.edu; stevezhanggeek@gmail.com; majid@cs.ucla.edu).

W. Xu is with the Department of Computer Science and Engineering, the State University of New York (SUNY) at Buffalo, NY 14260-1660, USA (e-mail: wenyaoxu@buffalo.edu).

Color versions of one or more of the figures in this paper are available online at http://ieeexplore.ieee.org.

Digital Object Identifier 10.1109/JBHI.2013.2296891 in order to check that the patients' recovery progresses according to plan. With manual monitoring, there is not only a large cost in tracking rehabilitation progress, but also the measurement of progress is difficult to quantize. Automated monitoring of rehabilitation exercises is proposed here as a solution.

\section{A. Related Work}

In this subsection, we describe the current literature in the field of automated physical rehabilitation monitoring. Zhou and Hu's survey [4] of human motion tracking systems for rehabilitation targeted primarily stroke sufferers, but this list of technological approaches can be applied to rehabilitation in general. Specialized exercise equipment, such as treadmills, or even robotic guidance devices [5] have been investigated for strength building applications. In addition, visual tracking of body posture has undergone much research, however many relied on marker systems placed on the body [6]. Furthermore, marker-free systems try to overcome these limitations by building 2-D [7] or 3-D models of the human body [8]. Other nonvisual methods have been researched, such as using inertial sensors [9]. Combination of sensors are also described, such as Huang's et al. work with inertial sensors and visual camera that tracked both (fine-grain) finger and (course-grain) hand movements for upper extremities rehabilitation [10]. These systems require extensive deployment or calibration and can be inconvenient for the users.

This paper focuses on rehabilitation monitoring of subject motions and posture changes, and in particular, with patients who are bed-ridden or restricted to motions on bed. There are current research approaches that focus on posture change detection, such as research by Nakajima et al. in which real-time video image sequences were used to extract optical flow information [11]. Jones et al. used a 24 pressure sensor array to identify posture movement times and then evaluated sleep restlessness [12], while Adami et al. used four load cell sensors with $200-\mathrm{Hz}$ sampling rates to analyze time varying waveforms as patients move on bed [13].

Accurate detection of posture movement are shown in these previous methods. They are able to show the existence of posture changes, but they do not target the recognition of actual posture. A system to detect transitions between sitting and lying postures using large pressure sensor arrays placed under the mattress was developed by Foubert et al. [14]. Yousefi et al. were successful in recognizing sleep posture using principal component analysis (PCA) [15], while Hsia et al. used statistical feature extraction [16]. Harada et al. investigated body posture tracking and used generative models of human body pressure to match the patient's pressure distribution [17]. Similarly, our 
Training Process

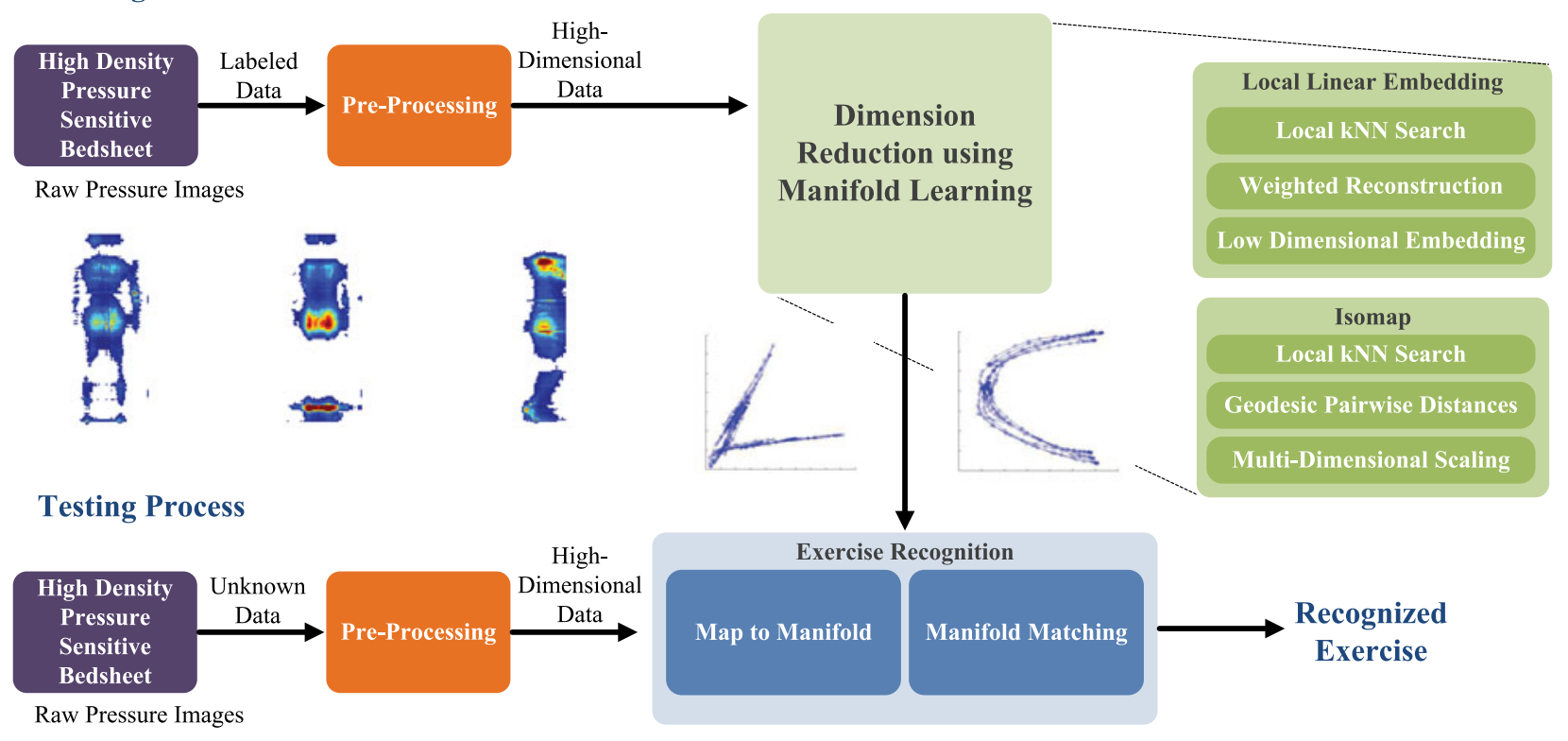

Fig. 1. Process flow for on-bed exercise monitoring.

previous work in this field investigated static posture recognition [18], however, this current paper focuses on the transitional and dynamic nature of body motion as seen in pressure image sequences. Most related studies used a very limited number of sensors for measuring pressure distribution and do not report any dynamic on-bed motion results. Therefore, we conclude that there are no similar type of on-bed exercise monitoring methods with which to compare our results.

\section{PRELIMINARIES}

\section{A. Bedsheet Design}

The bedsheet system consists of three components: a $64 \times$ 128 pressure sensor array, an embedded data sampling unit, and a tablet for data analysis and storage. The sensor array is based on an eTextile material which is fiber-based yarn coated with piezoelectric polymer [19], [20]. Without pressure, the resistance of the eTextile material is high. As external force is applied to the surfaces of the material, the eTextile fibers are squeezed together and, due to its pressure sensitive characteristics, the electrical resistance decreases in that region.

The bedsheet itself has a three-layer structure. The top layer is regular fabric that is coated with 64 parallel conductive lines. The middle layer is the eTextile material and the bottom layer has 128 conductive lines arranged perpendicular to the top 64 lines. At each intersection of conductive lines, the structure forms a pressure sensitive resistor. There are effectively 8192 pressure sensors in total.

\section{B. Manifold Structure of Pressure Image Sequences}

In general, image analysis on raw high dimensional data is computationally expensive, so dimension reduction techniques are usually applied to transform the data into another domain to either make it more manageable or useful for further analysis.

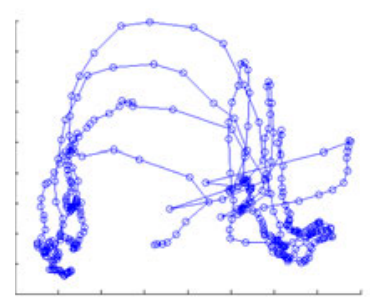

(a)

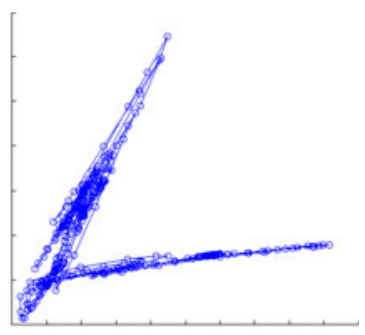

(b)
Fig. 2. Comparison visualization of Dimension Reduction to two dimensions. (a) PCA. (b) LLE manifold.

PCA is a well known method of dimension reduction and has widespread use in applications such as face recognition [21], speech analysis [22], and medical classification [23]. PCA finds the principal components, i.e., the main orthogonal base axes with which each data sample can be linearly constructed. The result is that only a subset of base axes are required to sufficiently represent the original data.

Fig. 2 is an example of the benefit of using manifold learning for this application. Compared with PCA, manifold learning is better suited to identify the underlying topological structure that is nonlinear in the high dimensional image space. Manifold learning approaches are also less prone to overfitting since the intrinsic dimensionality of manifolds is low (see Section IV-C for discussion on intrinsic dimensionality).

\section{Algorithm Overview}

Fig. 1 introduces the three main steps, and this process is described in more detail in the following section.

Step 1: Preprocessing of the pressure image

Step 2: Dimension reduction via manifold learning

Step 3: Activity recognition using manifold matching. 
Exercise recognition uses a subject's prerecorded training data to match exercises under test. The training data consists of samples of on-bed exercises that are analyzed to produce a low dimensional representation, i.e., a manifold, from the original high resolution pressure images. When new exercise data is recorded, it is mapped to the same low dimensional manifold representation and matched to the closest training exercise manifold.

\section{Algorithmic Framework For Exercise Monitoring}

- Step 1: Pre-processing

The pre-processing of the raw pressure images is required so that the image sequences can be standardized in such a way to enable successful recognition. The eTextile material exhibits voltage leakage across adjacent sensors. This causes noise and artifacts that affect recognition performance, and preprocessing mitigates these side effects as much as possible.

First, the subject can be located anywhere on the bedsheet, so to correct this, the images are aligned to a common center of mass and relocated to the center of the image. A smoothing filter of a symmetric $5 \times 5$ unit normal distribution is applied. This smoothing minimizes the effect of noise in the pressure map. The images are normalized so that the sum of pixel weights is one. This step attempts to counteract the effects for different body mass.

- Step 2: Dimension Reduction Using Manifold Learning

In this paper, we investigate two other methods of dimension reduction: local linear embedding (LLE) and Isomap. Both of these are manifold learning types of dimension reduction. The advantage of manifold learning techniques is that they can find the underlying structure, or the manifold, and can be visualized if the inherent dimension is low enough.

\section{A. Local Linear Embedding}

The first method to map the image sequence $X$ to a low dimensional space is based on the LLE framework by Saul and Roweis [24], which has various applications in machine learning systems [25]. LLE is an unsupervised algorithm that reconstructs the global data nonlinearly while preserving local linearity. After the computation, similar images will be clustered within the low dimensional manifold. In general, there are three steps in the algorithm, which will be described in the following.

1) k-Nearest Neighbor Searching: The first step is to search $\mathrm{k}$-nearest neighbors for each image. In the searching process, we use Euclidean distance to evaluate the similarity between images. For this paper, we searched for the 30 nearest neighbors of each image.

2) Weighted Reconstruction With Nearest Neighbors: The second step is to reconstruct a sample image using its nearest neighbors. Assume that an arbitrary image $x$ has k-nearest neighbors $x_{i}$. Then, $x$ can ideally be represented as a linear combination of its neighbors. In general, an exact reconstruction will not be found, so a reconstruction error $e$ for a single sample $x$ can be formulated as:

$$
e=\left\|x-\sum_{i=1}^{k} w_{i} x_{i}\right\|
$$

where $w_{i}$ denotes the reconstruction weight for the neighbor $x_{i}$.

For $N$ images, we can write the total error as:

$$
E=\sum_{j=1}^{N}\left\|x_{j}-\sum_{i=1}^{N} w_{i j} x_{i j}\right\|
$$

where each $x_{j}$ is an image. $x_{i j}$ is the $i$ th neighbor of $x_{j}$, and $w_{i j}$ is the reconstruction weight for $x_{i j}$. The optimization process minimizes the reconstruction error of all images by finding appropriate values for the weights $w_{i j}$. There are two attributes of the problem to ensure it is well-imposed: (1) exclusiveness: the weight $w_{i j}$ is zero if $x_{i j}$ is not in the nearest neighbor list of $x_{j}$; (2) normalization: the sum of the weights of nearest neighbors of $x_{j}$ should be 1. Equation (2) has a closed least square solution and the weights $w_{i j}$ can be solved efficiently [24].

3) Low Dimensional Embedding Construction: The third step is to construct the corresponding embedding in a low dimensional space. Based on the calculation results from the second step, the intrinsic geometrical structure of each local cluster is characterized by $w_{i j}$. We assume that the neighborhood relation in high dimensional space should be preserved in low dimensional space, i.e., within a manifold. Based on this assumption, the embedding process finds the low dimensional representation $y$ of $x$ by minimizing the following error $E^{\prime}$ :

$$
E^{\prime}=\sum_{j=1}^{N}\left\|y_{j}-\sum_{i=1}^{N} w_{i j} y_{i j}\right\|
$$

where $y_{j}$ and $y_{i j}$ are the corresponding points of $x_{j}$ and $x_{i j}$ in the low dimensional manifold, respectively. We note that (3) is in a quadratic form and the embedding optimization process is efficiently solvable. Furthermore, all the manifold points $y_{i}$ will be computed globally and simultaneously, and no local optima will affect the construction result.

Equations (2) and (3) indicate that the low dimensional construction is only based on the local neighborhood data. This means that the computed manifold $y_{i}$ can be translated with an arbitrary displacement. Moreover, LLE states the computed manifold $y_{i}$ can be rotated by an arbitrary angle without affecting (3) too. These geometric attributes can be represented and formulated with the following two constraints:

$$
\sum_{i=1}^{N} y_{i}=0, \quad \frac{1}{N} \sum_{i=1}^{N} y_{i} \cdot y_{i}=1 .
$$

Therefore, manifold construction problem becomes an eigenvalue problem [24], in which we select the matrix rank to have the desired manifold dimension.

\section{B. Isomap}

The second method to map the image sequence $X$ to a low dimensional space is based on the Isomap framework by Tenenbaum et al. [26]. Isomap also performs nonlinear dimension 
reduction and extends the classical linear Multidimensional Scaling (MDS) method [27]. MDS finds a set of co-ordinates that satisfies given pairwise distances between points. However, given that the points may lie on a manifold inside a high dimensional space, linear pairwise distances may not represent the true structure.

1) $k$-Nearest Neighbor Searching: This stage is similar to LLE where k-Nearest Neighbors for each original image are selected using the Euclidean distance in high dimensions.

2) Computation of Geodesic Pairwise Distance: The distances between all pairs of images are estimated given the distances between local sets of images. This results in the geodesic pairwise distances, i.e., the shortest path distances $D_{i j}^{X}$ along a curved surface. Floyd's algorithm can be used to find the shortest paths between every pair of images in a graph, or other faster methods [28].

3) Low Dimensional Embedding via Multidimensional Scaling: The final stage of Isomap applies the regular MDS algorithm to the geodesic distances. MDS finds points $Y$ that minimize the total error between pairwise distances in high dimensional space and pairwise distances in low dimensional space:

$$
\min _{Y} \sum_{i=1}^{N} \sum_{j=1}^{N}\left(D_{i j}^{X}-D_{i j}^{Y}\right)^{2}
$$

where $D_{i j}^{Y}$ is the pairwise distance between points in the low $d$ dimensional space $Y$. The low dimensional embedding is solved by taking the co-ordinates of the top $d$ eigenvectors of the innerproduct matrix of the geodesic pairwise distances [27].

Contrasting with LLE, Isomap preserves pairwise distances within the manifold, while LLE preserves the local linear structure within the manifold.

- Step 3: Exercise Recognition Using Manifold Matching

1) Map Input to Manifold: Once the training data has been reduced in dimensionality to its corresponding low dimensional form, we can evaluate the process using new test data against the training data. The testing data needs to be converted into manifold form. Note that it is possible to run the whole LLE or Isomap algorithm again on the combined testing data and training data in order to find the low dimensional representation of the test data, however this is not necessary.

Instead, a portion of the algorithm needs only to be executed [24], [29]. Given a new test image $\hat{x}$, we wish to find its low dimensional representation, $\hat{y}$. To do so, the weights $w_{i}$ are computed from the $\mathrm{k}$ nearest neighbors of $\hat{x}$ in the training set, $x_{i}$. This is again the least squares solution to minimize

$$
\left\|\hat{x}-\sum_{i=1}^{k} w_{i} x_{i}\right\|
$$

with the constraint $\sum_{i=1}^{k} w_{i}=1$. Since the corresponding low dimensional co-ordinates of $x_{i}$ are known during the training phase, we can construct the resultant embedded co-ordinates for $\hat{y}$ using the same weights:

$$
\hat{y}=\sum_{i=1}^{k} w_{i} y_{i}
$$

where $y_{i}$ are the corresponding embedded points of $x_{i}$.

2) Manifold Matching: Exercise tracking involves checking how well the testing data follows the trajectory of a given exercise manifold. We can compare trajectories using a similar idea to the Hausdorff distance. The distance of a point to a manifold is equal to the shortest Euclidean distance to any point in the manifold. The similarity of two manifolds is the mean of the point distances of all the points of one manifold, $M_{1}$ to the other manifold, $M_{2}$. This is expressed as

$$
s\left(M_{1}, M_{2}\right)=\frac{1}{T_{M_{1}}} \sum_{i=1}^{T_{M_{1}}} \min _{1 \leq j \leq T_{M_{2}}}\left\|M_{1}(i)-M_{2}(j)\right\|
$$

where $T_{M_{1}}$ and $T_{M_{2}}$ are the number of points in each manifold. This metric allows manifolds of different lengths to be compared since different subjects take different times to perform each activity. Since the Hausdorff metric is not symmetric, we can take the following sum as the manifold matching metric,

$$
d\left(M_{1}, M_{2}\right)=s\left(M_{1}, M_{2}\right)+s\left(M_{2}, M_{1}\right) .
$$

So, to measure how well a subject adheres to the prescribed exercise, the testing data is mapped to corresponding low dimensional embedding points that defines a manifold, then the manifold is measured against the expected exercise manifold.

\section{EXPERIMENTS AND DISCUSSION}

\section{A. Experimental Setup}

We evaluated this framework for exercise monitoring onten subjects, seven male subjects, and three female subjects. The weight of the subjects ranged from 50 to $85 \mathrm{~kg}$, and height between 155 and $188 \mathrm{~cm}$. There were five selected on-bed exercises: alternating leg lifts, head lifts, alternating heel slides, alternating side-to-side lateral rolls, and sit ups. These exercises have been selected as being appropriate for on-bed monitoring [30]. In the training data collection, at least five sets of image sequences were recorded for each of the five on-bed exercises for each subject, so there were more than 250 exercise sequences under test. Each image sequence comprises one exercise activity, e.g., one leg lift exercise activity includes lifting of the right leg followed by the left leg. The order of left and right does not matter in this system. Each image sequence of exercise activity contained at least 40 individual images. Small variations in body, arm, and leg positions were allowed for each subject since only gross motor functions would be analyzed. Examples of exercise and sample images are shown in Figs. 3 and 4.

The training data for each subject was combined and manifold learning was applied to generate the training manifolds for the exercises. Testing was carried out by exercise activity and repeated for each of the exercise activities. 


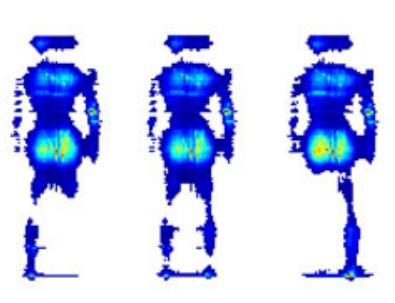

(a)

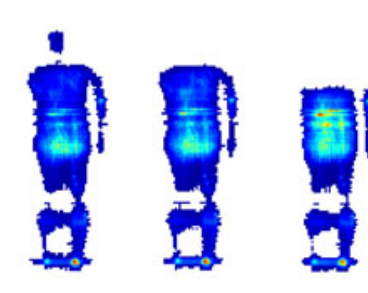

(b)
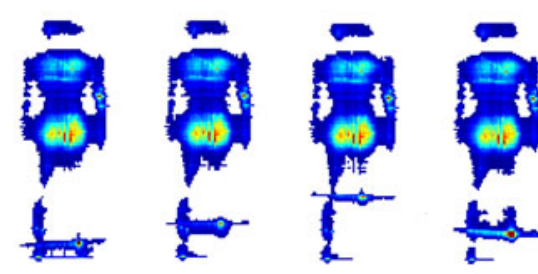

(c)
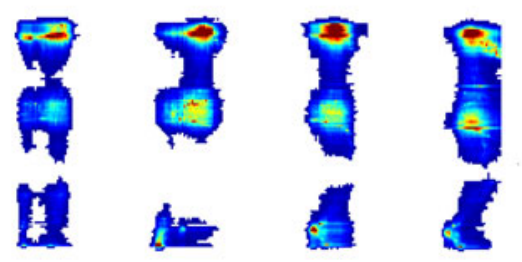

(d)
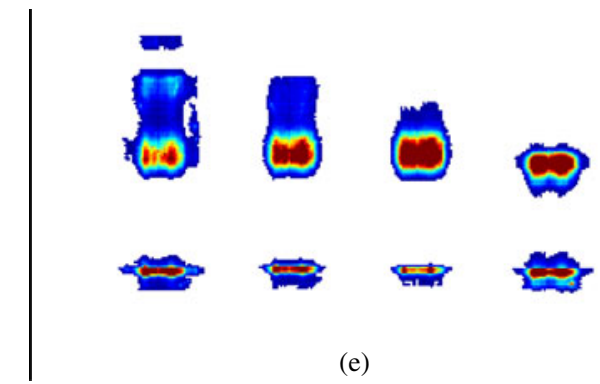

(e)

Fig. 3. Sample images. (a) Leg lift. (b) Head lift. (c) Heel slide. (d) Lateral roll. (e) Sit up.

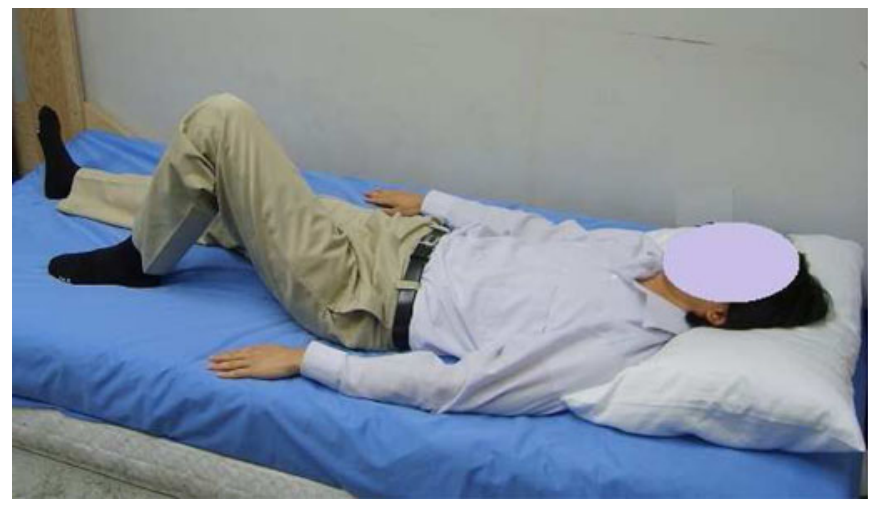

Fig. 4. Example of heel slide exercise.

\section{B. Experimental Evaluation}

Manifolds within high dimensional space exist because similar patterns that correspond to exercises trace similar paths. Distinguishing between different exercises is possible since the corresponding low dimensional manifold representations exhibit different paths. Due to the smooth body pressure transformations in the image sequences, manifold learning methods such as LLE and Isomap are able to produce low dimensional reconstructions of the data points. Manifold learning is well suited to this type of exercise recognition application because human body motion is somewhat limited on-bed and there are only slight variations in posture changes across different subjects.

Tables I and II show recognition results for the five exercises in ten subject dependent testing, i.e., subjects are not tested with training data from other subjects. Notably, the highest recognition rates are lateral rolls and sit ups. This can be expected since these exercises involve the greatest physical exertion and hence the greatest pressure image differences. The other three exercises exhibit a comparably lower rate of recognition due to more of a fine grain difference in the pressure image sequences.
TABLE I

CONFUSION MATRIX LLE

\begin{tabular}{|c|c|c|c|c|c|c|}
\hline & LL & HL & HS & LR & SU & Recall \\
\hline LL & 42 & 4 & 6 & 0 & 0 & $80.8 \%$ \\
HL & 9 & 42 & 2 & 0 & 0 & $79.2 \%$ \\
HS & 7 & 0 & 54 & 0 & 0 & $88.5 \%$ \\
LR & 0 & 0 & 0 & 50 & 0 & $100 \%$ \\
SU & 0 & 0 & 0 & 1 & 56 & $98.2 \%$ \\
\hline Precision & $72.4 \%$ & $91.3 \%$ & $87.1 \%$ & $98.0 \%$ & $100 \%$ & \\
\hline
\end{tabular}

TABLE II

CONFUSION MATRIX ISOMAP

\begin{tabular}{|c|c|c|c|c|c|c}
\hline & LL & HL & HS & LR & SU & Recall \\
\hline LL & 45 & 3 & 4 & 0 & 0 & $86.5 \%$ \\
HL & 7 & 44 & 2 & 0 & 0 & $83.0 \%$ \\
HS & 6 & 1 & 54 & 0 & 0 & $88.5 \%$ \\
LR & 0 & 0 & 0 & 49 & 1 & $98.0 \%$ \\
SU & 0 & 0 & 0 & 3 & 54 & $94.7 \%$ \\
\hline Precision & $77.6 \%$ & $91.7 \%$ & $90.0 \%$ & $94.2 \%$ & $98.2 \%$ & \\
\hline
\end{tabular}

The confusion matrix shows that there are the most misclassifications between leg lifts versus head lift, followed by leg lifts versus heel slides. The former observation is explained by similar muscular pressure that is required when lifting one's head and legs, in that more pressure is added in the back region. As to the latter, there are clear similarities in the pressure images of Fig. 3, and the leg positions only constitute minor variations in the overall pressure maps.

Fig. 5 shows samples of the low dimensional visualization of manifolds for some of the exercises. Generally, the shapes of the manifolds give an indication of the differences between the exercises. It is interesting to note in Fig. 5(d) that the variations in sit ups can be seen. Compared to leg lifting or rolling motions, the subjects showed a greater range of styles in the sit-up action. Some people could sit up directly with legs slightly bent and, later on, needed to rotate their body or use hands to facilitate the effort. 


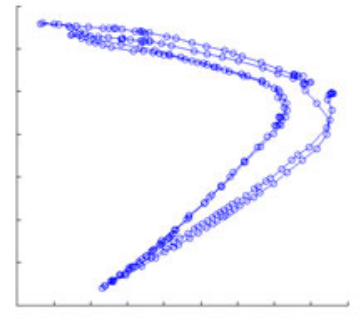

(a)

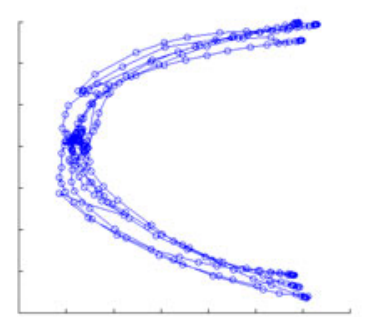

(c)

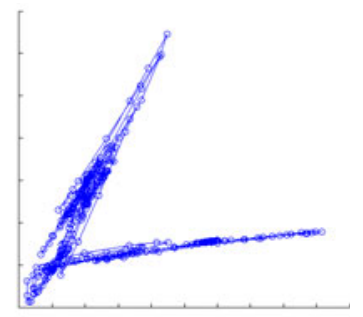

(b)

(d)

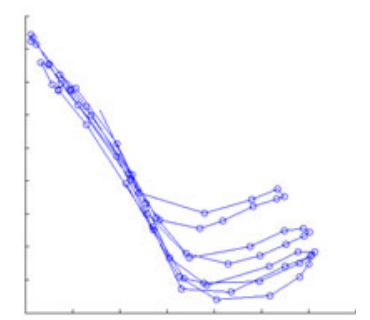

Fig. 5. Samples of exercise manifolds LLE. (a) Leg lift. (b) Heel slide. (c) Lateral roll. (d) Sit up.

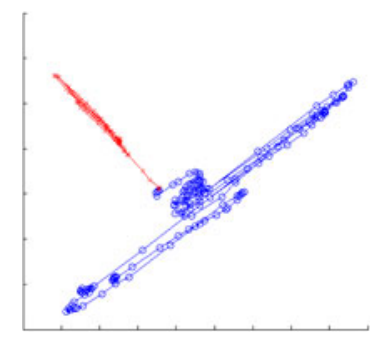

(a)

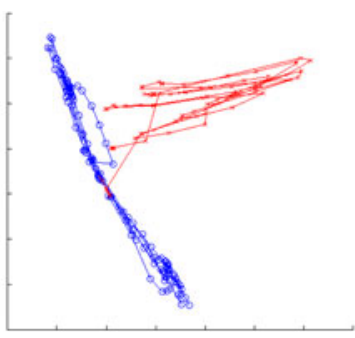

(b)
Fig. 6. Samples of exercise manifolds LLE. (a) Leg lift (blue) versus head lift (red). (b) Roll (blue) versus sit up (red).

\section{Comparing LLE and Isomap}

The accuracy results between LLE and Isomap are somewhat similar as shown in Tables I and II. LLE has higher accuracy for the lateral rolls and sit ups, while Isomap shows higher accuracy for leg lifts, head lifts, and heel slides. This suggests that LLE, since it uses linear structure, is able to differentiate larger motions, while Isomap, since it uses a distance based metric in the regions about each point of interest, is more suited to finer grained motions in these exercises.

Fig. 6 shows samples of how head lifts appear on a leg-lift manifold under LLE, and sit up compared to lateral rolls. Fig. 7 shows the same results for Isomap. It is evident that the sample exercises can be discerned from each other. It is interesting to note that the manifold shapes produced by Isomap tend to be more variable, which may contribute to the slight variation in accuracy levels.

The dimension reduction algorithm requires the data to be nonsparse, i.e., there must be sufficient sampling of pressure images to track motions. The current state of technology for pressure images of this resolution are 2-5 samples per second. Higher sampling rates can be achieved with the loss of image resolution, i.e., more images can be sampled per second but with lower pixel resolution.

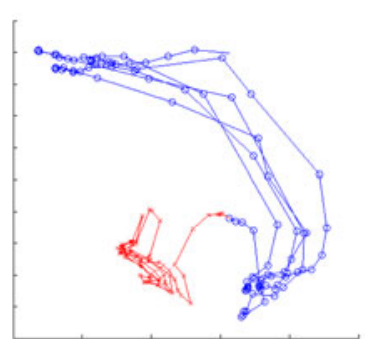

(a)

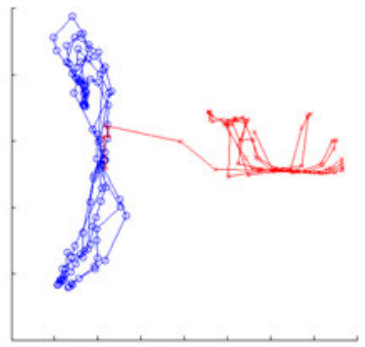

(b)
Fig. 7. Samples of exercise manifolds Isomap. (a) Leg lift (blue) versus head lift (red). (b) Roll (blue) versus sit up (red).

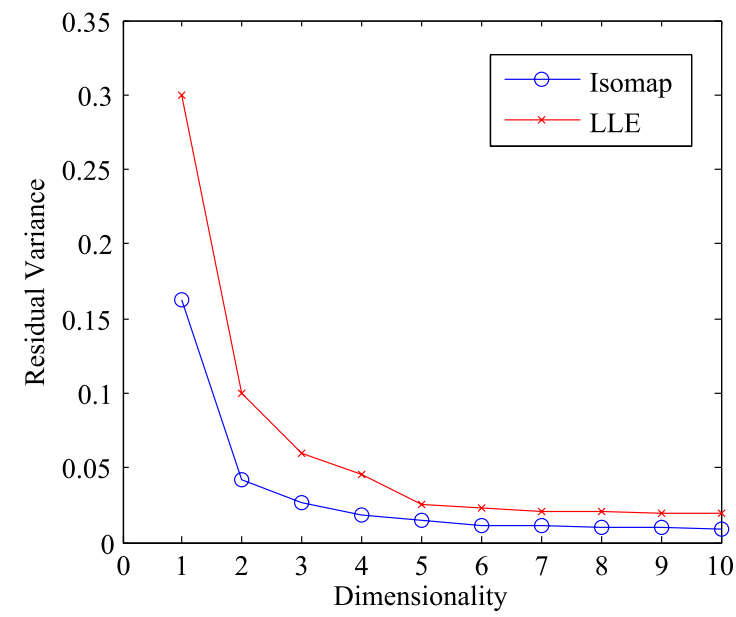

Fig. 8. Intrinsic dimensionality of the manifolds are found at the knee points of the graph.

To verify that manifold learning is an appropriate method for exercise recognition, we investigate the intrinsic dimension, i.e., how many variables are sufficient to describe the changes in the data. Fig. 8 suggests that the intrinsic dimension of the rehabilitation exercise data is 2 . In the dimensionality graph, this is seen at the knee point, i.e., where the curves have the largest break. As we reduce the dimensionality of the reconstruction space, the reconstruction error or residual variance slowly increases until dimension 2. A single dimension is clearly not sufficient as it produces large reconstruction errors.

\section{Sequential Evaluation}

As a further evaluation of this framework for exercise recognition, we investigated a longitudinal study of continuous monitoring through a number of set exercise activity programs. More specifically, each of the five exercises was performed sequentially to analyze whether the algorithm can separate and recognize the exercises. The order of exercises was chosen arbitrarily and it is not atypical in rehabilitation therapy. Fig. 9 shows the evaluation results of this experiment, where red dash line represents the ground truth, and the blue line represents the classification results. We can see that three sets of five different exercises are performed sequentially in this evaluation, and finally 13 out of the 15 exercises are recognized correctly. 


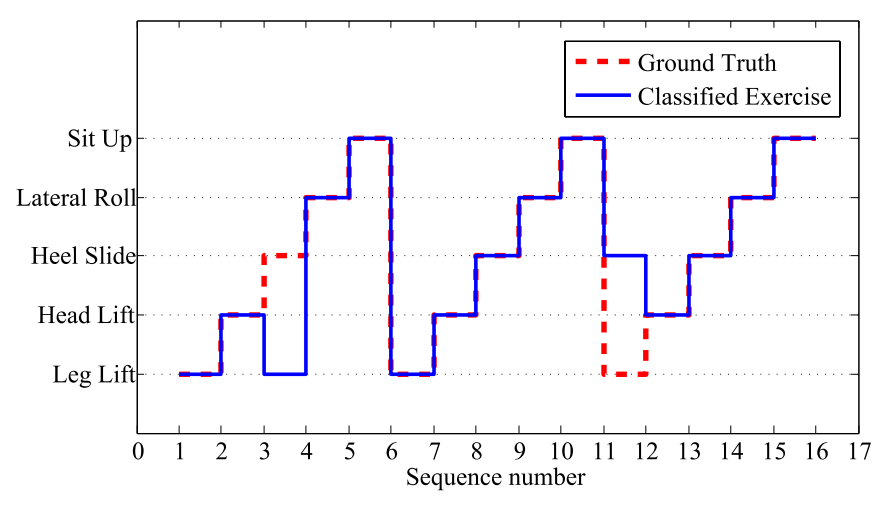

Fig. 9. Set of 15 exercise activities performed sequentially against ground truth.

\section{CONCLUSION}

In this paper, an on-bed exercise monitoring system design, that allows care-givers to track compliance to physical rehabilitation programs, is presented. We employ the novel use of dimension reduction techniques from pressure images to find intrinsic subspace representations of the data. We also evaluated a metric to match manifolds to enable quantified measurement of adherence to prescribed exercises.

Future work involves quantifying the performance of a given exercise with respect to standard exercise models, i.e., how closely is the patient following the exercise patterns such as angle of leg lifts and length of heel slide. Other future endeavors includes facilitating a system to work on chairs for sitting rehabilitative exercise, not only in clinical rooms or home-base care but also for cars or wheelchairs. 3-D model reconstruction of patients from 2-D pressure image is another goal that can be accomplished using the results of this research work.

\section{ACKNOWLEDGMENT}

The authors would like to thank Medisens Wireless Inc. for their contributions in discussion, building and supply of the hardware.

\section{REFERENCES}

[1] A. L. Behrman and S. J. Harkema, "Physical rehabilitation as an agent for recovery after spinal cord injury," Phys. Med. Rehab. Clin. North Amer., vol. 18, no. 2, pp. 183-202, 2007.

[2] M. Fransen, J. Crosbie, and J. Edmonds, "Physical therapy is effective for patients with osteoarthritis of the knee: A randomized controlled clinical trial," J. Rheumatol., vol. 28, no. 1, 2001.

[3] R. Lord, C. Mayhew, R. Korupolu, E. Mantheiy, M. Friedman, J. Palmer, and D. Needham, "ICU early physical rehabilitation programs: Financial modeling of cost savings," Crit. Care Med., Jan. 2013.

[4] H. Zhou and H. Hu, "Human motion tracking for rehabilitation-A survey," Biomed. Signal Proc. Contr., vol. 3, pp. 1-18, 2008.

[5] H. I. Krebs, B. T. Volpe, M. L. Aisen, and N. Hogan, "Increasing productivity and quality of care : Robot-aided neuro-rehabilitation," Rehabil. Res. Development, vol. 37, p. 639, Nov. 2000.

[6] Y. Tao and H. Hu, "Buiding a visual tracking system for home-based rehabilitation," in Proc. 9th Chinese Autom. Comput. Soc. Conf. U.K., 2003, pp. 343-348.

[7] R. Fablet and M. J. Black, "Automatic detection and tracking of human motion with a view-based representation," in Proc. 7th Eur. Conf. Comput. Vis.-Part I, 2002, pp. 476-491.
[8] Q. Delamarre and O. Faugeras, "3d articulated models and multiview tracking with physical forces," Comput. Vis. Image Understand., vol. 81, no. 3, pp. 328-357, 2001.

[9] E. Jovanov, A. Milenkovic, C. Otto, P. de Groen, B. Johnson, S. Warren, and G. Taibi, "A WBAN system for ambulatory monitoring of physical activity and health status: Applications and challenges," in Proc. 27th Annu. Int. Conf. Eng. Med. Biol. Soc., Jan. 2005, pp. 3810-3813.

[10] M.-C. Huang, W. Xu, Y. Su, B. Lange, C.-Y. Chang, and M. Sarrafzadeh, "Smartglove for upper extremities rehabilitative gaming assessment," in Proc. 5th Int. Conf. Pervas. Technol. Related Assist. Envir, 2012, pp. 20:120:4.

[11] K. Nakajima, Y. Matsumoto, and T. Tamura, "Development of real-time image sequence analysis for evaluating posture change and respiratory rate of subject in bed," Physiol. Meas., vol. 22, no. 3, 2001.

[12] M. Jones, R. Goubran, and F. Knoefel, "Identifying movement onset times for a bed-based pressure sensor array," in Proc. IEEE Med. Meas. Appl. Int. Workshop, Apr. 2006, p. 111.

[13] A. M. Adami, M. Pavel, T. L. Hayes, and C. M. Singer, "Detection of movement in bed using unobtrusive load cell sensors," Trans. Inf. Technol. Biomed., vol. 14, no. 2, p. 481, Mar. 2010.

[14] N. Foubert, A. McKee, R. Goubran, and F. Knoefel, "Lying and sitting posture recognition and transition detection using a pressure sensor array," in Proc. IEEE Int. Symp. Med. Meas. Appl., May 2012, pp. 1-6.

[15] R. Yousefi, S. Ostadabbas, M. Faezipour, M. Farshbaf, M. Nourani, L. Tamil, and M. Pompeo, "Bed posture classification for pressure ulcer prevention," in Proc. Eng. Med. Biol. Soc., Sep. 2011, p. 7175.

[16] C.-C. Hsia, Y.-W. Hung, Y.-H. Chiu, and C.-H. Kang, "Bayesian classification for bed posture detection based on kurtosis and skewness estimation," in Proc. e-health Network., Appl. Serv., HealthCom 10th Int. Conf., Jul. 2008, pp. 165-168.

[17] T. Harada, T. Sato, and T. Mori, "Human motion tracking system based on skeleton and surface integration model using pressure sensors distribution bed," in Proc. Workshop Human Motion, 2000, p. 99.

[18] J. J. Liu, W. Xu, M.-C. Huang, N. Alshurafa, and M. Sarrafzadeh, "A dense pressure sensitive bedsheet design for unobtrusive sleep posture monitoring," in Proc. IEEE Int. Conf. Pervas. Comput. Commun., Mar. 2013, pp. 207-215.

[19] W. Xu, Z. Li, M.-C. Huang, N. Amini, and M. Sarrafzadeh, "eCushion An eTextile device for sitting posture monitoring," in Proc. Body Sensor Networks (BSN), May. 2011, pp. 194-199.

[20] W. Xu, M.-C. Huang, N. Amini, J. J. Liu, L. He, and M. Sarrafzadeh, "Smart insole: A wearable system for gait analysis," in Proc. 5th Int. Conf. Pervas. Tech. Relat. Assist. Env., 2012, pp. 18:1-4.

[21] J. Yang, D. Zhang, A. Frangi, and J.-Y. Yang, "Two-dimensional pca: a new approach to appearance-based face representation and recognition," IEEE Trans. Pattern Anal. Mach. Intell., vol. 26, no. 1, pp. 131-137, Jan. 2004.

[22] S. Wang, X. Ling, F. Zhang, and J. Tong, "Speech emotion recognition based on principal component analysis and back propagation neural network," in Proc. Int. Conf. Meas. Technol. Mechatron. Autom., 2010, vol. 3, pp. 437-440.

[23] X. Long, B. Yin, and R. Aarts, "Single-accelerometer-based daily physica activity classification," in Proc. IEEE Annu. Int. Conf. Eng. Med. Biol. Soc., 2009, pp. 6107-6110.

[24] L. Saul and S. Roweis, "Think globally, fit locally: Unsupervised learning of low dimensional manifolds," J. Mach. Learn. Res., vol. 4, pp. 119-155, Dec. 2003.

[25] Z. Li, W. Xu, A. Huang, and M. Sarrafzadeh, "Dimensionality reduction for anomaly detection in electrocardiography: A manifold approach," in Proc. 9th Int. Conf. Wearable Implant. Body Sens. Netw., 2012, pp. 161165.

[26] J. B. Tenenbaum, V. d. Silva, and J. C. Langford, "A global geometric framework for nonlinear dimensionality reduction," vol. 290, no. 5500 pp. 2319-2323, 2000.

[27] J. Kruskal and M. Wish, Multidimensional Scaling, (Sage University Paper series on Quantitative Applications in the Social Sciences, 07-011). Beverly Hills, CA, USA: Sage Publications, 1978.

[28] V. Kumar, A. Grama, A. Gupta, and G. Karypis, Introduction to Parallel Computing: Design and Analysis of Algorithms. Redwood City, CA, USA: Benjamin-Cummings Publishing Co., Inc., 1994.

[29] M. Zhang and A. Sawchuk, "Manifold learning and recognition of human activity using body-area sensors," in Proc. 10th Int. Conf. Mach. Learn. Apps Workshop, Dec. 2011, vol. 2, pp. 7-13.

[30] S. Nettina and L. W. Wilkins, The Lippincott Manual of Nursing Practice. Philadelphia, PN, USA: Lippincott Williams \& Wilkins, 2006. 


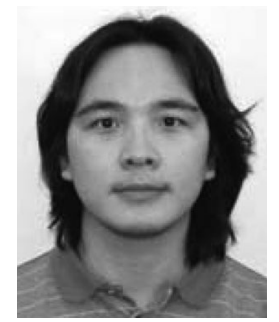

Ming-Chun Huang (S'11) received the B.S. degree in computer science from National Tsing Hua University, Taiwan, and the M.S. degree in electrical engineering from University of Southern California, Los Angeles, CA, USA. He is currently working toward the $\mathrm{Ph} . \mathrm{D}$. degree in computer science at the University of California, Los Angeles, CA, USA.

His research interests are in the area of medical sensor system design, computational modeling, and motivation-driven research, named data networking, and applications of smart infrastructure design.

Mr. Huang won the Best Medical \& Performance Application Paper Award of IEEE Conference on Implantable and Wearable Body Sensor Networks, in 2013, and the Best Demonstration Award in ACM Wireless Health Conference in 2011 . He currently holds five patents.

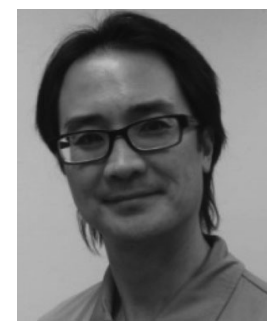

Jason J. Liu (S'10) received the B.Sc. degree in computer science and the B.E. degree in electrical engineering with first class honors from the University of Sydney, Sydney, Australia. He received the M.S.E. degree in robotics from the University of Pennsylvania, Philadelphia, PA, USA, and is currently working toward the Ph.D. degree in computer science at the University of California, Los Angeles, CA, USA.

His research interests include computer vision, machine learning, medical informatics, wireless health, and human-computer interaction.

Mr. Liu received a Best Paper Award at the IEEE Conference on Implantable and Wearable Body Sensor Networks in 2013.

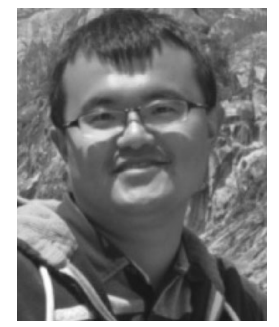

Wenyao Xu (M'13) received the Ph.D. degree from the Department of Electrical Engineering, University of California, Los Angeles, CA, USA, in 2013.

Currently, he is an Assistant Professor with the Department of Computer Science and Engineering, University at Buffalo, the State University of New York (SUNY), New York, NY, USA. His current research interests include embedded system design, biomedical signal processing, human computer interaction, integrated circuit design technologies, and their applications in medical and healthcare domains.

Dr. Xu received the Best Paper Award of the IEEE Conference on Implantable and Wearable Body Sensor Networks in 2013 and the Best Demonstration Award of ACM Wireless Health Conference in 2011. He holds five international/US patents.

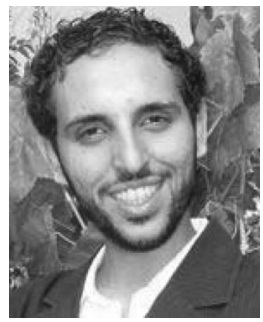

Nabil Alshurafa (S'13) received the B.Sc. degree in computer science, graduating summa cum laude from the University of California, Los Angeles, CA, USA, in 2003. He received the M.Sc. degree in computer science with an emphasis on artificial life, robotics and global optimization, in 2010, and is currently working toward the Ph.D. degree in computer science at the University of California, Los Angeles.

He served for eight years as a Project Manager and Software Developer in the field of signal processing, feature extraction, and classification for object recognition and target tracking, and was awarded several government grants. His current research interests include signal processing for activity recognition, machine learning, prediction and modeling in wireless health, and system optimization for remote health monitoring.

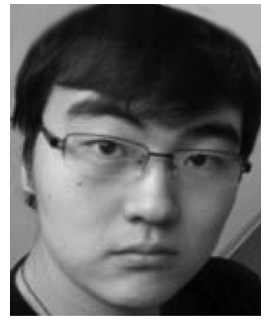

Xiaoyi Zhang (S'13) is currently an undergraduate student with the Department of Computer Science, University of California, Los Angeles, CA, USA. His research interests are in the area of body sensor network, wireless health, data visualization, computer vision applications, and human-computer interaction. He has interned at Apple and Google Research. $\mathrm{He}$ is also a creative Project Leader in Hackathon.

In LA Hacks, Mr. Zhang won Best Overall, Mobile App \& Data Visualization. He won second place in Facebook Hackathon. In HackUCLA, he won Grand Prize \& Tech Difficulty Prize.

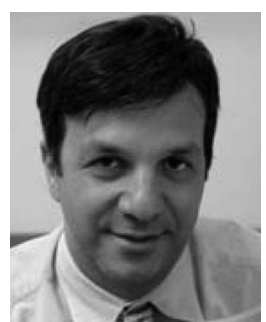

Majid Sarrafzadeh (F'96) received the Ph.D. degree in electrical and computer engineering from the University of Illinois, Urbana-Champaign, IL, USA, in 1987.

He joined Northwestern University, Evanston, IL, USA, as an Assistant Professor in 1987. In 2000, he joined the Department of Computer Science, University of California, LA, USA. He is a Co-Founder and a Co-Director of the UCLA Wireless Health Institute. His current research interests include embedded computing with emphasis on healthcare. He has published over 400 papers, co-authored five books, and is a named inventor on many U.S. patents. He has collaborated with many industries in the past 25 years. $\mathrm{He}$ co-founded two companies during 2000 - they were both acquired around 2004. $\mathrm{He}$ has recently co-founded MediSens and Bruin Biometrics, both in the area of Technology in Healthcare. 